\title{
Corrigendum: Inonotus sanghuang Polyphenols Attenuate Inflammatory Response Via Modulating the Crosstalk Between Macrophages and Adipocytes
}

\section{OPEN ACCESS}

Edited and reviewed by: Willem Van Eden, Utrecht University, Netherlands

${ }^{*}$ Correspondence: Junpeng Wang jpwangchina@henu.edu.cn

${ }^{t}$ These authors share senior authorship

Specialty section: This article was submitted to Nutritional Immunology, a section of the journal

Frontiers in Immunology

Received: 25 November 2020 Accepted: 05 January 2021 Published: 28 January 2021

Citation:

Zhang $M$, Xie $Y$,

Su X, Liu K, Zhang $Y$, Pang $W$ and Wang J (2021) Corrigendum: Inonotus sanghuang Polyphenols Attenuate Inflammatory Response Via Modulating the Crosstalk Between Macrophages and Adipocytes.

Front. Immunol. 12:633354. doi: 10.3389/fimmu.2021.633354

\author{
Mengdi Zhang ${ }^{1}$, Yu Xie ${ }^{1,2}$, Xing Su ${ }^{1}$, Kun Liu ${ }^{3}$, Yijie Zhang ${ }^{1 \dagger}$, Wuyan Pang ${ }^{1 \dagger}$ \\ and Junpeng Wang ${ }^{\text {* }}$

\begin{abstract}
${ }^{1}$ Institute of Infection and Immunity of Huaihe Hospital, Henan University, Kaifeng, China, ${ }^{2}$ School of Physical Education, Henan University, Kaifeng, China, ${ }^{3}$ College of Biology Science and Engineering, Hebei University of Economics and Business, Shijiazhuang, Hebei, China
\end{abstract}

Keywords: Inonotus sanghuang, polyphenols, inflammation, obesity, NF-KB signaling, MAPK signaling

\section{A Corrigendum on}

Inonotus sanghuang Polyphenols Attenuate Inflammatory Response Via Modulating the Crosstalk Between Macrophages and Adipocytes

By Zhang, M., Xie, Y., Su, X., Liu, K., Zhang, Y., Pang, W., et al. (2019). Front. Immunol. 10:286. doi: 10.3389/fimmu.2019.00286

In the original article, there was a mistake in the total STAT3 expression of western blotting data in Figure 7B as published. There was an unintentional error in the Figure 7B. The corrected Figure 7 appears below.

The authors apologize for this error and state that this does not change the scientific conclusions of the article in any way. The original article has been updated.

Copyright $\odot 2021$ Zhang, Xie, Su, Liu, Zhang, Pang and Wang. This is an open-access article distributed under the terms of the Creative Commons Attribution License (CC BY). The use, distribution or reproduction in other forums is permitted, provided the original author(s) and the copyright owner(s) are credited and that the original publication in this journal is cited, in accordance with accepted academic practice. No use, distribution or reproduction is permitted which does not comply with these terms. 
A

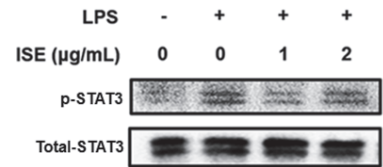

$\underset{\substack{\text { LPS } \\ \text { ISE (Ng/mL) }}}{0}$

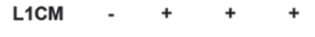

ISE $(\mu \mathrm{g} / \mathrm{mL}) \quad 0 \quad 0 \quad 1 \quad 2$

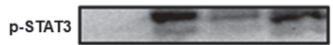

Total-STAT3 $=\equiv$

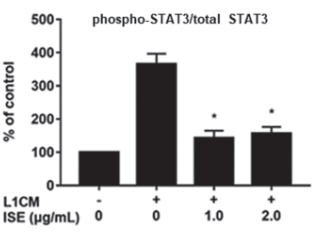

FIGURE 7 | Effects of ISE on STAT3 signal. RAW264.7 macrophages were pretreated with different concentrations of ISE for $1 \mathrm{~h}$ and then stimulated by $1.0 \mu \mathrm{g} / \mathrm{mL}$ LPS or 3T3-L1CM for 30min. Total cell lysates were extracted, and then western blotting using specific antibodies was used to determine the expression of p-STAT3 and STAT3 in LPS (A) and 3T3-L1CM (L1CM) stimulation (B), respectively. The value of a control was set at 100\%, and the relative value was presented as fold induction to that of the control, which was normalized to total STAT3. Statistical comparisons were made with each vehicle controls. The values are means \pm SD, $n=3 .{ }^{*} P<0.05,{ }^{\star \star} P<0.01$. 